\title{
Complications and Mishaps in Anesthesia. Cases - Analysis - Preventive Strategies
}

\author{
Matthias Hübler, Thea Koch, Karen B. Domino (Editors). Springer, 2014, \$79.99, \\ paperback, 365 pages. ISBN 978-3-642-45406-6
}

\author{
Daniel Dubois, MD
}

Received: 3 December 2014/ Accepted: 9 December 2014/Published online: 24 December 2014

(C) Canadian Anesthesiologists' Society 2014

The first English-language edition of this paperback book is comprised of 33 chapters with 365 pages (including 46 illustrations). It is dedicated to outlining complications related to anesthesia and concepts of the humanistic side of medicine. The authors have produced a narrative consistent with a 'case-based learning' approach to analyze and develop preventive strategies for reducing the number of medical errors. The book engages the reader by using humour and real-life situations frequently encountered in everyday practice.

The narrative follows the interaction between allied health workers in a wide range of anesthesia-related scenarios encountered in the clinic, operating theatre, and recovery room. Embedded within each scenario is background information on up-to-date medical knowledge or skills pertaining to crisis resource management. Each chapter is organized into three parts: a case described in a short, light-hearted vignette; a clinically relevant surgical/ medical problem; and a review of factors that might have led to a significant error. The information is delivered in a concise, engaging style, offering medical knowledge embedded in the pragmatic content. Practical tips from the authors provide valuable insights into behavioural and situational awareness. The most relevant part of each case is the conclusion, wherein the authors provide a short debriefing that outlines potential errors that may have led to a mishap. The discussion points related to possible errors touch on communication, adequate supervision of trainees, and the interaction between judgement, motivation, and emotion that informed the decision-making processes. In real life, a misstep frequently involves a psychological

D. Dubois, MD ( $\square)$

Health Sciences Centre, Winnipeg, MB, Canada

e-mail: danielduboismd@gmail.com precursor (distraction, preoccupation, forgetfulness, fatigue, stress) that is often the last link in a chain of events leading to an accident or adverse event. Throughout the chapters, the authors emphasise that accidents are rarely the result of a single unsafe act. Rather, they are most often the product of many factors, including situational, organizational, equipment-related, and behavioural missteps.

Karen Domino's updated and translated edition includes sufficient references, including many new review articles pertaining to medical management. The authors make use of clear diagrams to complement and help explain the text. I found the range of general anesthesia knowledge in each chapter to be of wide breadth, although succinct.

My criticisms are minor. I did not agree with the University Hospital of Dresdens' unexpected difficult airway algorithm. I found it disordered, and it contradicted the American Society of Anesthesiologists difficult airway algorithm by failing to mention the option of returning to spontaneous ventilation or awakening the patient. In addition, their algorithm for failed mask ventilation/intubation suggested the use of more muscle relaxation. Furthermore, after failed laryngeal mask airway (LMA) insertion, it was suggested that direct laryngoscopy followed by another trial of LMA insertion should be attempted.

Although several texts provide either medical knowledge or a review of errors in medicine, this book attempts to link the two elements in an easily digestible format. It is insightful, reflective, and a valuable read for anyone looking to minimize errors. The book brings to light common pitfalls that we encounter in our everyday practice and complements other traditional textbooks on the subject of medical errors and patient safety. The subject matter and anesthesia pearls covered in this book will be of 
great relevance to anesthesiologists at all levels of training. It provides a good starting point for teaching anesthesia trainees and could prompt targeted discussion. I wholeheartedly recommend this book to trainees and clinicians involved in perioperative care. The short anecdotal format is a comfortable method for review and makes for enjoyable ten-minute sessions. It would be an excellent light read during a busy operating room day.

Conflicts of interest None declared. 